\title{
Neutral Gas Outside the Disks of Local Group Galaxies
}

\author{
Felix J. Lockman \\ National Radio Astronomy Observatory $\dagger$ \\ Green Bank National Observatory \\ P.O. Box 2 \\ Green Bank, WV 24944 USA \\ email: jlockman@nrao.edu
}

\begin{abstract}
Of the three kinds of neutral gas found outside the stellar disks of Local Group galaxies, only the products of interaction, like the Magellanic Stream, have a clearly understandable origin. Both the high-velocity clouds and the faint H I between M31 and M33 remain a mystery. New observations of the region between M31 and M33 with the Green Bank Telescope show that the H I there resides in clouds with a size and mass similar to that of dwarf galaxies, but without stars. These clouds might be products of an interaction, or condensations in the hot circumgalactic medium of M31, but both these models have difficulties. The prevalence of clouds like this in the Local Group remains to be determined.
\end{abstract}

Keywords. Galaxy: evolution, Galaxy: formation, Galaxy: halo, galaxies: Local Group

\section{Introduction}

Although the neutral atomic Hydrogen in the Local Group of galaxies is overwhelmingly concentrated in the galaxies, significant $\mathrm{HI}$ is found far beyond the scale-height expected for gas in equilibrium with a galaxy's potential:

High Velocity Clouds: From the earliest surveys in the $21 \mathrm{~cm}$ line of neutral hydrogen, there was clear evidence of gas at high latitudes that deviated from Galactic rotation by $\gtrsim 100 \mathrm{~km} \mathrm{~s}^{-1}$, the high-velocity clouds (HVCs) (Muller et al. 1963). This material covers about $40 \%$ of the sky in the $21 \mathrm{~cm}$ line and perhaps twice that area in warm ionized gas (Lockman et al. 2002, Shull et al. 2009, Lehner \& Howk 2011). Despite significant progress in determining the distance, physical properties, and even abundances and ionization stage of many HVCs, and despite the fact that they are now observed around other galaxies (e.g., M31 Thilker et al. 2004) their origin is still unknown (Oort 1966, Wakker \& van Woerden 1997, Putman et al. 2012).

Products of Interaction: The clearest example is the Magellanic Stream, the result of the interaction between the Magellanic Clouds and the Milky Way (Wannier \& Wrixon 1972, Mathewson et al. 1974, Putman et al. 2003, Besla 2015). It stretches more than $200^{\circ}$ across the sky and contains at least $0.5 \times 10^{9} \mathrm{M}_{\odot}$ in $\mathrm{HI}$ and $1.5 \times 10^{9} \mathrm{M}_{\odot}$ in $\mathrm{H}^{+}$ (Nidever et al. 2010, Fox et al. 2014). The starbursting dwarf galaxy IC10 may be another example of an interaction in progress (Nidever et al. 2013).

Inter-group gas? The discovery by Braun \& Thilker (2004, hereafter BT04) of regions of extremely low surface-brightness H I emission around M31 extending toward the galaxy M33 raised the possibility that there might be a significant amount of neutral gas that was, to some extent, "free-floating" in the Local Group. BT04 suggested that this material

$\dagger$ The National Radio Astronomy Observatory is a facility of the National Science Foundation, operated under cooperative agreement by Associated Universities, Inc. 


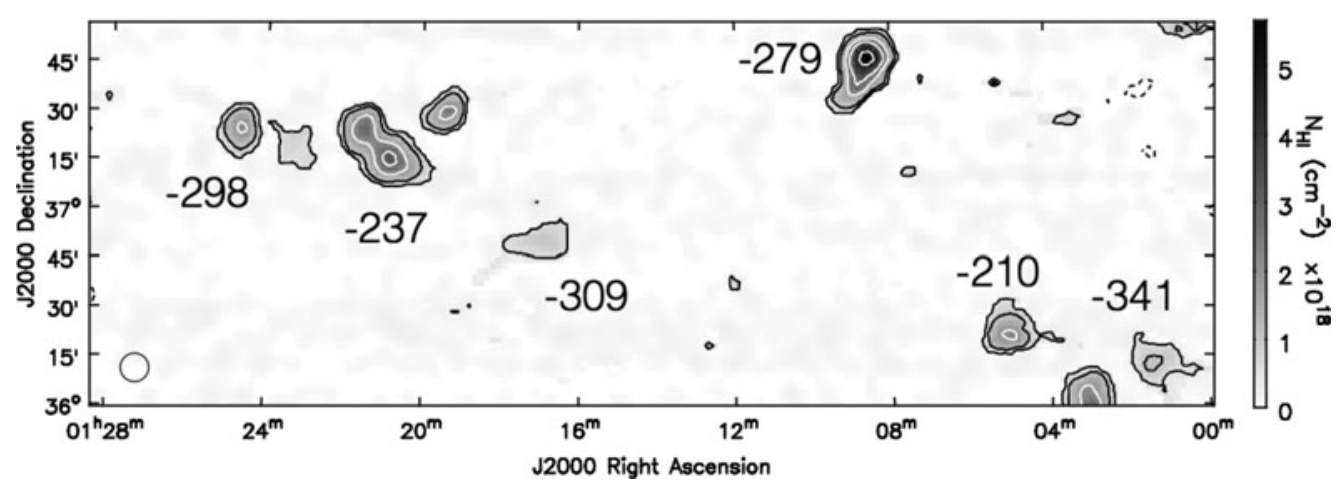

Figure 1. Discrete HI clouds detected between M31 and M33 with the GBT (from Wolfe et al. 2016). M31 lies off this figure to the upper right; M33 to the lower left. Contours show values of $\mathrm{N}_{\mathrm{HI}}$ in increments of $5 \times 10^{17} \mathrm{~cm}^{-2}$. Several clouds are marked with their $\mathrm{V}_{\mathrm{LSR}}$. The clouds appear to be internally coherent, yet there are large differences in velocity from one cloud to another, indicating that they are not only spatially isolated, but kinematically isolated structures as well. They are thus not likely to be regions of enhanced density in a larger object.

had condensed from a dark-matter filament connecting M31 with M33, and there were proposals that it resulted from a past interaction between M31 and M33 (e.g., Bekki 2008, Lewis et al. 2013). Recent $21 \mathrm{~cm}$ observations have revealed new properties of the $\mathrm{HI}$ and limit scenarios for its origin.

\section{GBT Observations of the HI between M31 and M33}

Taking advantage of the superb sensitivity to faint $21 \mathrm{~cm}$ H I emission provided by the Robert C. Byrd Green Bank Telescope (GBT), our group has been mapping regions around M31 at $9^{\prime}$ angular resolution to $\sigma_{\mathrm{NHI}}=9 \times 10^{16} \mathrm{~cm}^{-2}$ (Wolfe et al. 2013, Wolfe et al. 2016). We find that the $\mathrm{HI}$ in a $6^{\circ} \times 2^{\circ}$ region centered approximately between M31 and M33 is resolved into discrete clouds that have a typical size of a few kpc and a typical $\mathrm{M}_{\mathrm{HI}}$ of $10^{5} \mathrm{M}_{\odot}$, assuming a distance of $800 \mathrm{kpc}$. Figure 1 shows the location of these clouds. Several are marked with their $\mathrm{V}_{\mathrm{LSR}}$. The field is $\sim 100 \mathrm{kpc}$ from the center of M31, and the total H I mass is $1.4 \times 10^{6} \mathrm{M}_{\odot}$.

The clouds have velocities inconsistent with the velocities of the HVC systems of M31 and M33, and do not seem to be a simple extension of the M31 HVC population, which is confined to $\sim 50 \mathrm{kpc}$ of M31 (Westmeier et al. 2008). Individually, the clouds have an H I mass and size similar to that of some dwarf galaxies, but do not appear to be associated with stellar systems. Given their linewidth and size, their virial mass is three orders of magnitude larger than the observed HI mass, so that without some other significant mass component they would be quite transient. The clouds share some properties of the Ultra-compact HVCs (Adams et al. 2013) but little is known about those objects.

As mentioned above, it has been suggested that the H I between M31 and M33 might have arisen from a past encounter beween these galaxies, but the most recent calculations indicate that they have never been closer to each other than they are now, making this explanation unlikely (Shaya \& Tully 2013). The clouds could be in pressure equilibrium with the massive M31 circumgalactic medium recently proposed by Lehner et al. 2015 . Indeed, there is enough mass in that model at a radius of $100 \mathrm{kpc}$ to provide all the observed H I even if the medium is $>90 \%$ ionized. The question then becomes whether these clouds are relatively common in the circumgalactic media of spirals like M31, or whether there is something special about the M31-M33 direction that has caused their 
formation. One difficulty with this interpretation is that there seems to be a discrepancy between the velocities of the clouds and that of M31's rotational velocity at their position. Unlike the M31 HVCs, which seem to have some connection, however qualitative, with the rotation of the M31 disk (Thilker et al. 2004), and unlike some Galactic HVCs, whose motion is nearly aligned with Galactic rotation (Lockman et al. 2002), the M31-M33 clouds are not so clearly connected kinematically with the rotation of nearby galaxies.

\section{The Extent of the Cloud Population}

Studies of the outskirts of galaxies in UV absorption lines find neutral gas out to impact parameters of at least $100 \mathrm{kpc}$ (e.g., Nielsen et al. 2013). While $21 \mathrm{~cm}$ emission measurements cannot reach the low columns accessible through UV lines, the GBT data suggest that very sensitive radio measurements like the ones presented here - more than an order of magnitude more sensitive than the limits of $10^{19} \mathrm{~cm}^{-2}$ to $10^{20} \mathrm{~cm}^{-2}$ reached is most surveys of galaxies (e.g., Heald et al. 2011, Walter et al. 2008) - may reveal previously unknown objects. Additional GBT observations are underway to the north of the field in Fig. 1 to determine if the cloud population is confined to the M31-M33 axis, or is more widely distributed throughout the Local Group. Spectroscopy of the clouds in species that would reveal their abundances and ionization stage would go a long way towards solving the puzzle of their origin.

\section{References}

Adams, E. A. K., Giovanelli, R., \& Haynes, M. P. 2013, ApJ, 768, 77

Bekki, K. 2008, MNRAS, 390, L24

Besla, G. 2015, arXiv:1511.03346

Braun, R. \& Thilker, D. A. 2004, A\&A A, 417, 421

Fox, A. J., Wakker, B. P., Barger, K. A., et al. 2014, ApJ, 787, 147

Heald, G., Józsa, G., Serra, P., et al. 2011, A\& A, 526, A118

Lehner, N. \& Howk, J. C. 2011, Science, 334, 955

Lehner, N., Howk, J. C., \& Wakker, B. P. 2015, ApJ, 804, 79

Lewis, G. F., Braun, R., McConnachie, A. W., et al. 2013, ApJ, 763, 4

Lockman, F. J., Murphy, E. M., Petty-Powell, S., \& Urick, V. J. 2002, ApJ, 140, 331

Lockman, F. J., Benjamin, R. A., Heroux, A. J., \& Langston, G. I. 2008, ApJ, 679, L21

Mathewson, D. S., Cleary, M. N., \& Murray, J. D. 1974, ApJ, 190, 291

Muller, C. A., Oort, J. H., \& Raimond, E. 1963, Acad. Sci. Paris Comptes Rendus, 257, 1661

Nidever, D. L., Majewski, S. R., Butler Burton, W., \& Nigra, L. 2010, ApJ, 723, 1618

Nidever, D. L., Ashley, T., Slater, C. T., et al. 2013, ApJ, 779, L15

Nielsen, N. M., Churchill, C. W., \& Kacprzak, G. G. 2013, ApJ, 776, 115

Oort, J. H. 1966, Bulletin of the Astronomical Institutes of the Netherlands, 18, 421

Putman, M. E., Staveley-Smith, L., Freeman, K. C., et al. 2003, ApJ, 586, 170

Putman, M. E., Peek, J. E. G., \& Joung, M. R. 2012, Ann. Rev. Astr. Ap., 50, 491

Shaya, E. J. \& Tully, R. B. 2013, MNRAS, 436, 2096

Shull, J. M., Jones, J. R., Danforth, C. W., \& Collins, J. A. 2009, ApJ, 699, 754

Thilker, D. A., Braun, R., Walterbos, R. A. M., et al. 2004, ApJ, 601, L39

Wakker, B. P. \& van Woerden, H. 1997, ARA\&A, 35, 217

Walter, F., Brinks, E., de Blok, W. J. G., et al. 2008, AJ, 136, 2563-2647

Wannier, P. \& Wrixon, G. T. 1972, ApJ, 173, L119

Westmeier, T., Brüns, C., \& Kerp, J. 2008, MNRAS, 390, 1691

Wolfe, S. A., Pisano, D. J., Lockman, F. J., et al. 2013, Nature, 497, 224

Wolfe, S. A., Lockman, F. J., \& Pisano, D. J. 2016, ApJ, 816, 81 